\title{
A new technique for the histological diagnosis of Paget's disease of the breast using a semiautomated core needle biopsy with a 14-gauge needle
}

\author{
Jacopo Nori ${ }^{1}$ - Giulia Bicchierai ${ }^{1}$ (D ) Francesco Amato ${ }^{2}$. Diego De Benedetto ${ }^{1}$. Cecilia Boeri ${ }^{1}$. Ermanno Vanzi ${ }^{1}$. \\ Federica Di Naro ${ }^{1}$. Simonetta Bianchi ${ }^{3}$. Vittorio Miele ${ }^{4}$
}

Received: 30 November 2020 / Accepted: 14 April 2021 / Published online: 26 April 2021

(C) Italian Society of Medical Radiology 2021

\begin{abstract}
Purpose The aim of our work is to illustrate a new technique for the histological diagnosis of Paget's disease (PD) using a core needle biopsy with a semiautomated 14-gauge needle called nipple-core needle biopsy (N-CNB). We report 3 years' experience in our senology unit.

Materials and methods Twenty-six women with 26 clinical of nipple-areola complex (NAC) changes with suspected PD and subjected to core needle biopsy using our new $14 \mathrm{G}$ semiautomated needle technique were included in our study group. Institutional review board approval was obtained for this retrospective analysis. A semiautomated biopsy gun with a 14-gauge, 15-cm-long needle was used for this new procedure. After a subcutaneous injection of anesthetic and spray-ice application to the NAC, the 14G needle was opened with the cradle exposed and positioned on the NAC with considerable pressure exerted on the same. The cradle was then closed by triggering the needle spring, and 2-4 core samples were withdrawn by moving the needle position each time. Clinical, instrumental and histological differences between the lesions that gave benign results after N-CNB and those that resulted PD were analyzed by applying the Fisher's exact test.

Results After N-CNB, 13/26 lesions were found to be PD (50\%) while 13/26 alterations were benign (50\%). No malignant lesions were detected during the follow-up in patients with benign N-CNB results. The diagnosis of PD obtained with N-CNB was confirmed in all 13 cases by means of a histological analysis of the surgical specimens. No significant post-biopsy complications were recorded. Patients with PD more frequently presented nipple retraction $(\rho=0.0407)$ and associated suspicious (i.e., BI-RADS 4 and 5) mammographic ( $\rho=0.0006)$ findings compared to patients whose N-CNB had given benign results and the difference was statistically significant.

Conclusions In conclusion, with this novel technique, we were able to obtain an easy, painless, major complication-free and accurate diagnosis of PD of the NAC using a semiautomated core needle biopsy with a 14-gauge needle.
\end{abstract}

Keywords Paget's disease $\cdot$ Breast $\cdot$ Nipple-areola complex $\cdot$ Core needle biopsy

\section{Introduction}

Giulia Bicchierai

giulia.bicchierai@gmail.com

1 Diagnostic Senology Unit, Azienda OspedalieroUniversitaria Careggi, Largo G. A. Brambilla 3, 50134 Florence, Italy

2 Radiology Department, Ospedale San Giovanni Di Dio, Agrigento, Italy

3 Division of Pathological Anatomy, University of Florence, Florence, Italy

4 Emergency Radiology Department, Azienda Ospedaliero-Universitaria Careggi, Florence, Italy
Paget's disease (PD) of the breast is a disorder of the nipple-areola complex (NAC) with an incidence of $1-3 \%$ of all breast carcinomas. Pathologic findings consist of characteristic large round or ovoidal Paget tumor cells with glandular features inside the nipple epidermis with disrupted tight junctions among keratinocytes and reactive changes in the dermis (plasma cell infiltration, hyperemia and serous exudation) which give rise to the characteristic clinical appearance [1-3].

In most cases (67-100\%), PD is associated with an underlying ductal carcinoma and in some cases with a lobular 
carcinoma, however, on rare occasions it may also be present without any underlying neoplasia, found in no more than $8 \%$ of patients with PD. The underlying carcinoma is usually central and mainly located near the areola, but it can also be located in any part of the breast and is multifocal or multicentric in about $32-41 \%$ of patients [4-6].

The clinical aspect of NAC can be variable; it is generally characterized by nipple or areolar erythema, eczema, ulceration and itching. The nipple may be retracted or hyperpigmented and with a bloody discharge. Lesions are almost always unilateral but because of its features, PD can be mistaken for a benign dermatologic disease involving the nipple such as dermatitis, thus delaying the diagnosis often by more than 12 months.

Alterations of the NAC may or may not be associated with a palpable mass in the same breast; if this is present there is a greater probability of having associated invasive carcinomas and lymph node involvement with a worse prognosis $[1,4,6,7]$.

PD therapy is still under debate; the therapeutic gold standard for these patients was initially considered mastectomy with or without axillary dissection, but later studies published in the literature on conservative surgery have shown long-term survival rates similar to mastectomy, and breast conservation with radiation therapy is currently considered an oncologically safe option. However, as this option may be compromised by the aforementioned diagnostic delays, in PD it is fundamental to be able to obtain an early correct histological diagnosis [2, 8-10].

The histological diagnosis of PD can be performed by incisional or non-incisional techniques such as scrape cytology, punch biopsy and incisional biopsy. While incisional biopsy is more invasive, expensive and at times cosmetically disfiguring, non-incisional techniques have variable rates of false positives for benign lesions and fail to obtain underlying breast tissue, and scrape cytology requires an experienced cytopathologist [1, 2, 11-13].

A single work published in the literature described a nipple-core biopsy technique using an automated biopsy gun (Bard Magnum ${ }^{\mathrm{TM}}$ Biopsy instrument, BD, Becton, Dickinson and Company, Franklin Lakes, USA) with a 14-Gauge needle in 40 patients. In this work the histological diagnosis was obtained in all cases [14].

The aim of our work is to illustrate a new technique for the histological diagnosis of PD using a core needle biopsy with a semiautomated biopsy gun (Precisa, Hospital Service, Rome, Italy) and a 14-gauge needle called Nipple-Core Needle Biopsy (N-CNB). We report 3 years' experience in our senology unit.

\section{Materials and methods}

We retrospectively collected data from our records on 5760 core needle biopsies (CNBs) performed between November 2016 and November 2019 at our Diagnostic Senology Unit. All patients signed informed consent forms for the procedures. Institutional review board (IRB) approval was obtained for this retrospective analysis, which was performed in a large university-referral hospital for breast disease. We retrospectively selected $28 \mathrm{~N}-\mathrm{CNBs}$ performed in 28 patients with clinical changes in NAC with suspected PD.

The inclusion criteria for the study were: clinical changes in NAC with suspected PD, patients with clinical eczema who failed to respond to hydrocortisone in 3-4 weeks, CNB of the NAC performed using our new semiautomated 14-gauge (14G) needle technique (N-CNB), conventional imaging (CI): Digital Mammography (DM), Digital Breast Tomosynthesis (DBT) and ultrasound (US) performed prior to the biopsy, CNB or vacuum-assisted breast biopsy (VABB) of BI-RADS4 or 5 lesions identified with DM in patients with suspected PD, DBT and US performed in our center, breast surgery performed in our center with availability of complete histological reports of CNB and surgical specimens (SS). Of the 28 patients, we excluded 2 who were referred to other institutions. Therefore, 26 women aged 17-86 years, mean age 58.2 years (standard deviation [SD] 15.8) with 26 clinical changes of NAC and suspected PD subjected to core needle biopsy using our new $14 \mathrm{G}$ needle technique were included in our study group.

Before the biopsy, all patients underwent a DM, DBT and US in our department to evaluate the presence of breast lesions associated with clinical findings at the NAC level. Initial DM and DBT studies were performed using a full-field digital mammography unit with tomosynthesis (Selenia Dimensions, Hologic, Bedford, USA). Hand held bilateral whole-breast US exams were conducted by one of six radiologists in our Senology Unit using a 10-13 MHz transducer and a US unit (ESAOTE, MyLab $70 \mathrm{XVG}$, Genoa, Italy), and prior to the US, the same radiologists also carried out a clinical exam of both breasts. All six radiologists, who had 8-30 years' experience in all breast imaging methods, also performed the biopsies. In their reports they specified the clinical findings identified at the NAC level, the possible presence of discharge, and any additional findings identified with US, DM or DBT classified according to the BI-RADS criteria, the presence of a palpable mass in the same breast as the NAC alteration. They also indicated the breast density evaluated on DM according to the BI-RADS criteria [15], as well as the personal history of breast cancer (BC) for each 
patient. The additional lesions identified with US, DM or DBT and classified as BI-RADS 4 or 5, were subjected to either CNB or VABB by the same radiologists. Patients diagnosed with PD performed contrast-enhanced magnetic resonance imaging (CE-MRI) or a contrast-enhanced mammography (CEM) as preoperative staging. Surgical excisions were subsequently carried out within 30 days after diagnosis.

One of the breast radiologists in the department then consulted our database in order to evaluate the follow-up examinations performed by the patients included in the study in the months following the biopsies, as well as to record any complications emerging after the biopsy, i.e., profuse bleeding, infection, severe pain.

\section{Description of the procedure}

A semiautomated biopsy gun (Precisa, Hospital Service, Rome, Italy) with a 14-gauge, 15-cm-long needle was used for this new procedure.

Before the biopsy, each patient was questioned about the existence of any allergies to anesthetic drugs and the possible use of anticoagulant/antiplatelet drugs, as happens before each breast biopsy procedure [16]. Prior to the biopsy the skin was disinfected with a $10 \%$ povidone iodine cutaneous solution (Poviderm, Nuova Farmec S.r.l., Settimo di Pescantina (VR), Italy) followed by a subcutaneous injection of $10 \mathrm{cc}$ of lidocaine hydrochloride $20 \mathrm{mg} / \mathrm{ml}$ (Fisiopharma S.r.l., Palomonte (SA)-ITALY) beneath the NAC. Five minutes after the injection, spray ice (Frigofast, FarmacZabban, Calderara Di Reno Bologna, Italy) was also applied to the NAC to increase the local analgesic effect. Figure 1.

The 14G needle was opened with the cradle exposed and then positioned on the NAC at the level of the skin alterations with a considerable pressure exerted (Figs. 2 and 3).

The cradle was then closed by triggering the needle spring and 2-4 core samples (range 1-5) were withdrawn per lesion by moving the needle position each time to sample different parts of the NAC (Figs. 3 and 4).

The material collected was placed in containers with $10 \mathrm{ml}$ of formalin $8 \%$ and sent to the pathological anatomy department together with the clinical-instrumental data of each patient for a histopathological analysis. After the biopsy, a simple patch was applied or surgical glue and a patch in case of profuse bleeding; a bag of instant ice was placed over the patch for 15 min during which the patient remained under observation. Following, the patient was able to leave the department with the recommendation to remove the patch $24 \mathrm{~h}$ after the biopsy.

The samples and surgical specimens of all the lesions were reviewed by two pathologists with more than 25 years' experience in breast pathology. Their reports included the presence of PD, benign skin changes and in case of

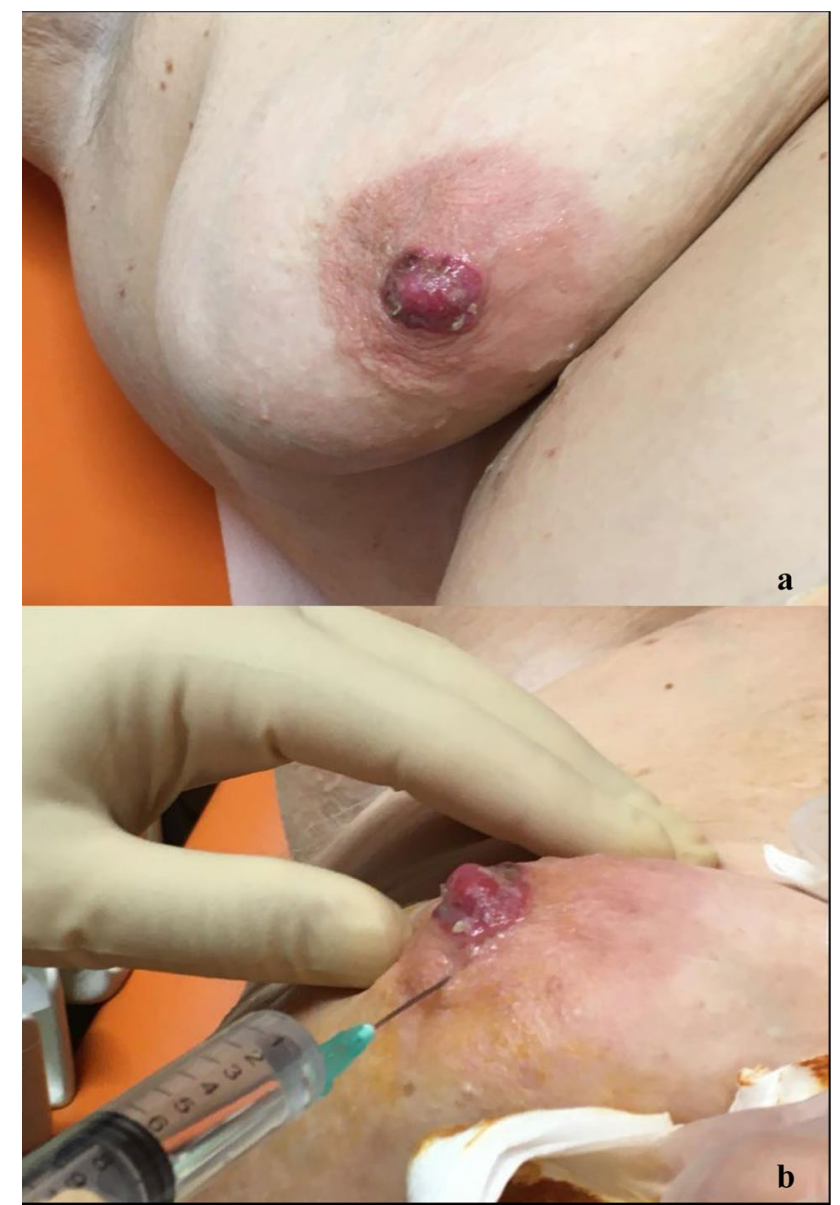

Fig. 1 a Suspicious clinical changes of the nipple-areola complex underwent our new semiautomated 14-gauge needle technique; b Infiltration of $10 \mathrm{cc}$ of lidocaine hydrochloride $20 \mathrm{mg} / \mathrm{ml}$ beneath the nipple-areola complex

associated carcinomas, the type, grade, size of tumor, estrogen receptor (ER) status, progesterone receptor $(\mathrm{PgR})$ status, human epidermal growth receptor factor 2 (HER2), Ki67 proliferative index, molecular subtypes and lymph node status [17]. The correlation between the imaging results and pathology for each case and the therapy-management decisions were discussed at the weekly multidisciplinary breast meeting.

\section{Statistical analysis}

Depending on the histological result of the biopsy, all the lesions included in the study and subjected to N-CNB were divided into benign lesions (B2) and PD lesions. The histologic results obtained from the surgically excised $\mathrm{PD}$ lesions were considered the gold standard for comparison with N-CNB findings. A negative follow-up was instead considered the gold standard for lesions found to be benign after N-CNB. 


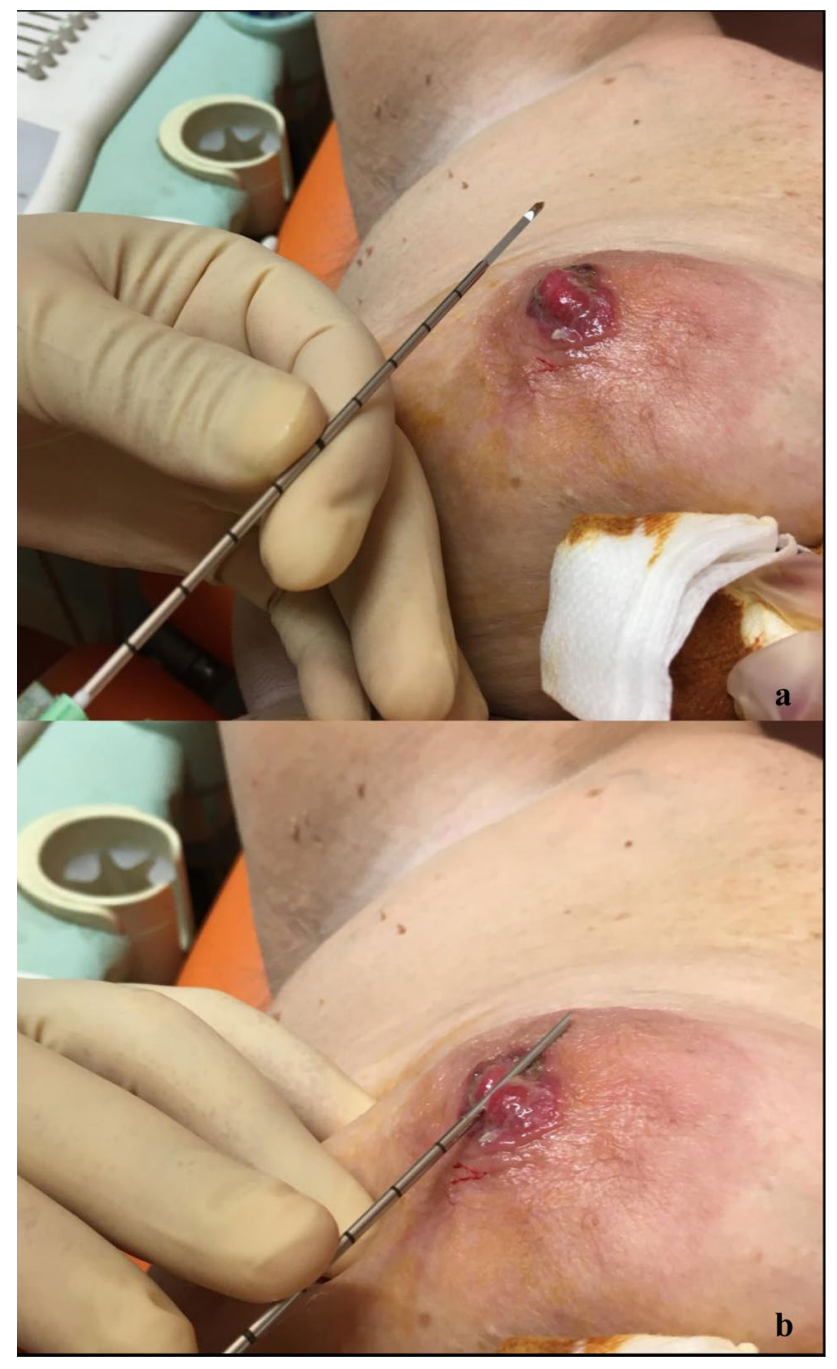

Fig. 2 a $14 \mathrm{G}$ needle is opened with the cradle exposed; b 14G needle with the cradle exposed is positioned on the suspicious skin alterations of the nipple-areola complex and considerable pressure exerted

Clinical, instrumental and histological differences between these two subgroups (i.e., benign lesions and PD lesions) were analyzed by applying the Fisher's exact test. A $\rho$ value $<0.05$ was considered to be statistically significant.

All the analyses were performed with IBM SPSS Statistics 23.0 (IBM SPSS Inc) and Microsoft Excel (Microsoft Corporation).

\section{Results}

Our study group consisted of 26 women aged $17-86$ years, mean age 58.2 years (standard deviation [SD] 15.8) with 26 clinical changes of NAC with suspected PD or clinical eczema that failed to respond to hydrocortisone in

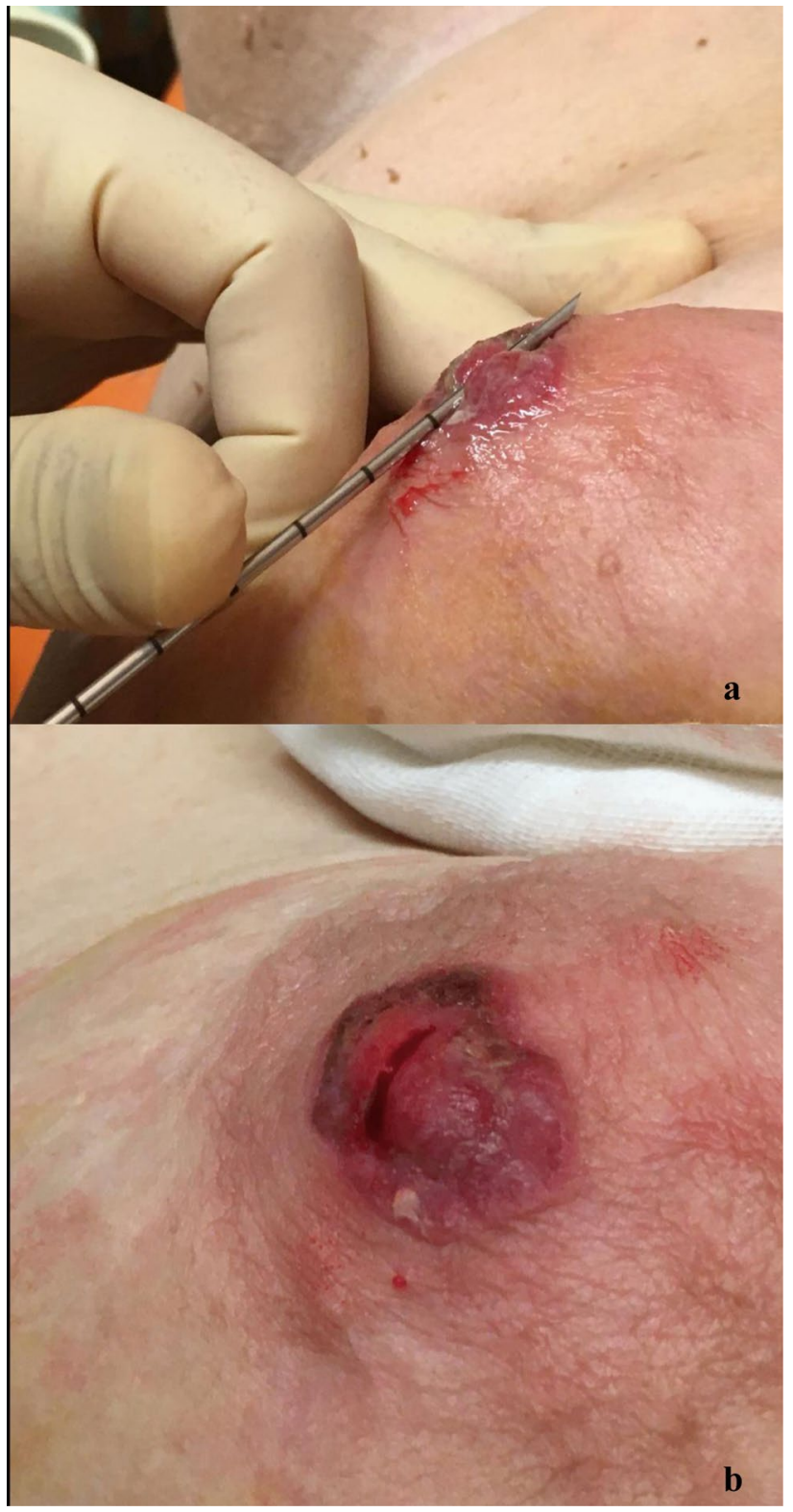

Fig. 3 a $14 \mathrm{G}$ needle with the cradle exposed is positioned on the suspicious skin alterations of the nipple-areola complex and considerable pressure exerted, detail. b How the nipple appears after the needle cradle has been closed and the sample removed

3-4 weeks, subjected to core needle biopsy using our new $14 \mathrm{G}$ needle technique ( $\mathrm{N}-\mathrm{CNB})$.

The characteristics of all the lesions included in the study are summarized in Table 1.

A histological diagnosis was obtained in all 26 cases. After N-CNB, 13/26 lesions were found to be PD (50\%) while $13 / 26$ alterations were benign $(50 \%)$. The followup time until now of all 26 lesions ranged between 13 and 53 months (average, 33 months). The average follow-up time of the benign lesions with $\mathrm{N}-\mathrm{CNB}$ was 30.9 months (range 18-49). All patients with benign results underwent an initial 


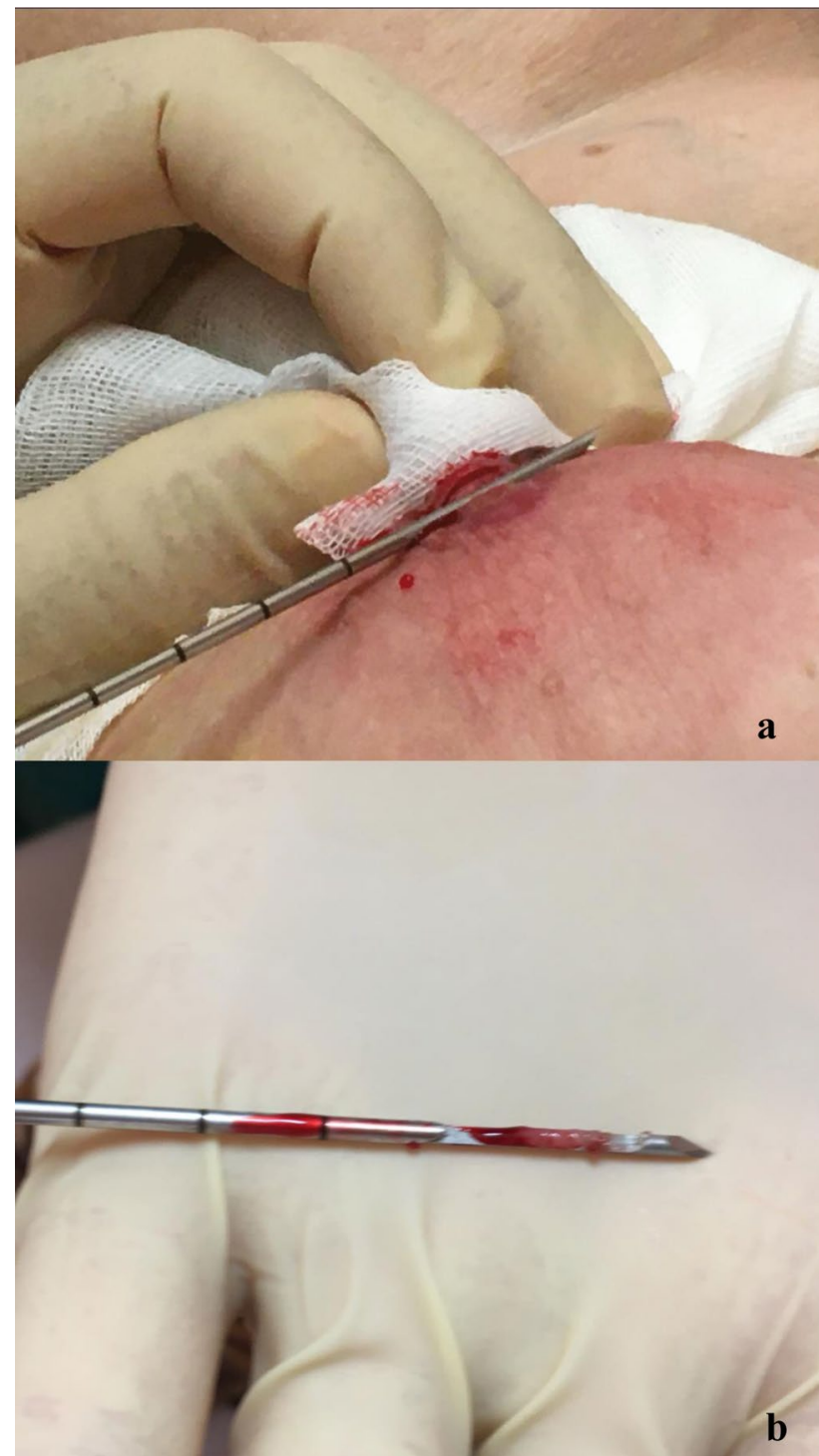

Fig. 4 a Biopsy is then repeated by positioning the $14 \mathrm{G}$ needle in a different point of the nipple. b Sample withdrawn with our core needle biopsy with a $14 \mathrm{G}$ semiautomated biopsy gun (N-CNB) technique, inside the open needle cradle

checkup with a physical examination, DM, DBT and US after six months, then follow-ups once a year in our department during which no malignant lesions were detected in any patients.

During the follow-ups of the 13 patients with PD, one with PD associated with invasive ductal carcinoma (IDC) developed liver metastases 12 months after the N-CNB treated with chemotherapy, and another with PD and DCIS developed an IDC in the contralateral breast after 46 months; this was one of the patients who refused to perform CEM or CE-MRI prior to surgery.

Of the 13 patients found to be affected by PD with N-CNB, 3/13 (23.1\%) had an associated clinically palpable
Table 1 Breast and lesion characteristics of the 26 women included in our study group with 26 suspicious clinical changes of the nippleareola complex (NAC) subjected to core needle biopsy using our new semiautomated $14 \mathrm{G}$ needle technique $(\mathrm{N}-\mathrm{CNB})$

\begin{tabular}{lr}
\hline Characteristic & \multicolumn{1}{c}{ Value } \\
\hline Nipple secretion & \\
Present & $9(34.6 \%)$ \\
Absent & $17(65.4 \%)$ \\
Nipple retraction & \\
Present & $4(15.4 \%)$ \\
Absent & $22(84.6 \%)$ \\
Mammography/DBT & \\
BI-RADS1;2;3 & $19(73.1 \%)$ \\
BI-RADS 4;5 & $7(26.9 \%)$ \\
Ultrasound & \\
BI-RADS1;2;3 & $23(88.5 \%)$ \\
BI-RADS 4;5 & $3(11.5 \%)$ \\
N-CNB results & \\
Paget & $13(50.0 \%)$ \\
Nipple adenoma & $3(11.5 \%)$ \\
Eczema & $3(11.5 \%)$ \\
Other benign lesions & $7(27.0 \%)$ \\
Breast density & \\
BI-RADS A & $2(7.7 \%)$ \\
BI-RADS B & $12(46.1 \%)$ \\
BI-RADS C & $6(23.1 \%)$ \\
BI-RADS D & $6(23.1 \%)$ \\
History of breast surgery & \\
Present & $6(23.1 \%)$ \\
Absent & $20(76.9 \%)$ \\
\hline
\end{tabular}

DBT Digital breast tomosynthesis

mass in the same breast, 4/13 (30.8\%) also had nipple discharge ( 3 bloody and one serous), and in $4 / 13$ (30.8\%) the nipple was retracted. Two patients of the 13 patients with $\mathrm{PD}$ had a history of breast cancer, 11 did not.

Of the 13 patients with PD, 4/13 (30.8\%) performed a CNB on BI-RADS 4 or 5 lesions identified with US, and 3/13 (23.1\%) a VABB on BI-RADS 4 or 5 lesions identified with DM DBT in addition to the N-CNB. Malignant lesions were detected in all these biopsies, 5/7 (71.4\%) were invasive carcinomas and 2/7 (28.6\%) ductal carcinoma in situ (DCIS), with six cases located in the same breast as the PD, and one in the contralateral breast. The characteristics of PD-associated malignant lesions, identified with US, DBT and DM, are summarized in Table 2.

Preoperative CEM was performed in 6/13 (46.1\%) patients after biopsy and 3/13 (23.1\%) underwent CE-MRI. These contrast-enhanced examinations demonstrated an abnormal nipple enhancement in six cases and the presence of additional suspicious enhancement areas (masses or nonmass enhancements classified as BI-RADS4 or 5) at least 


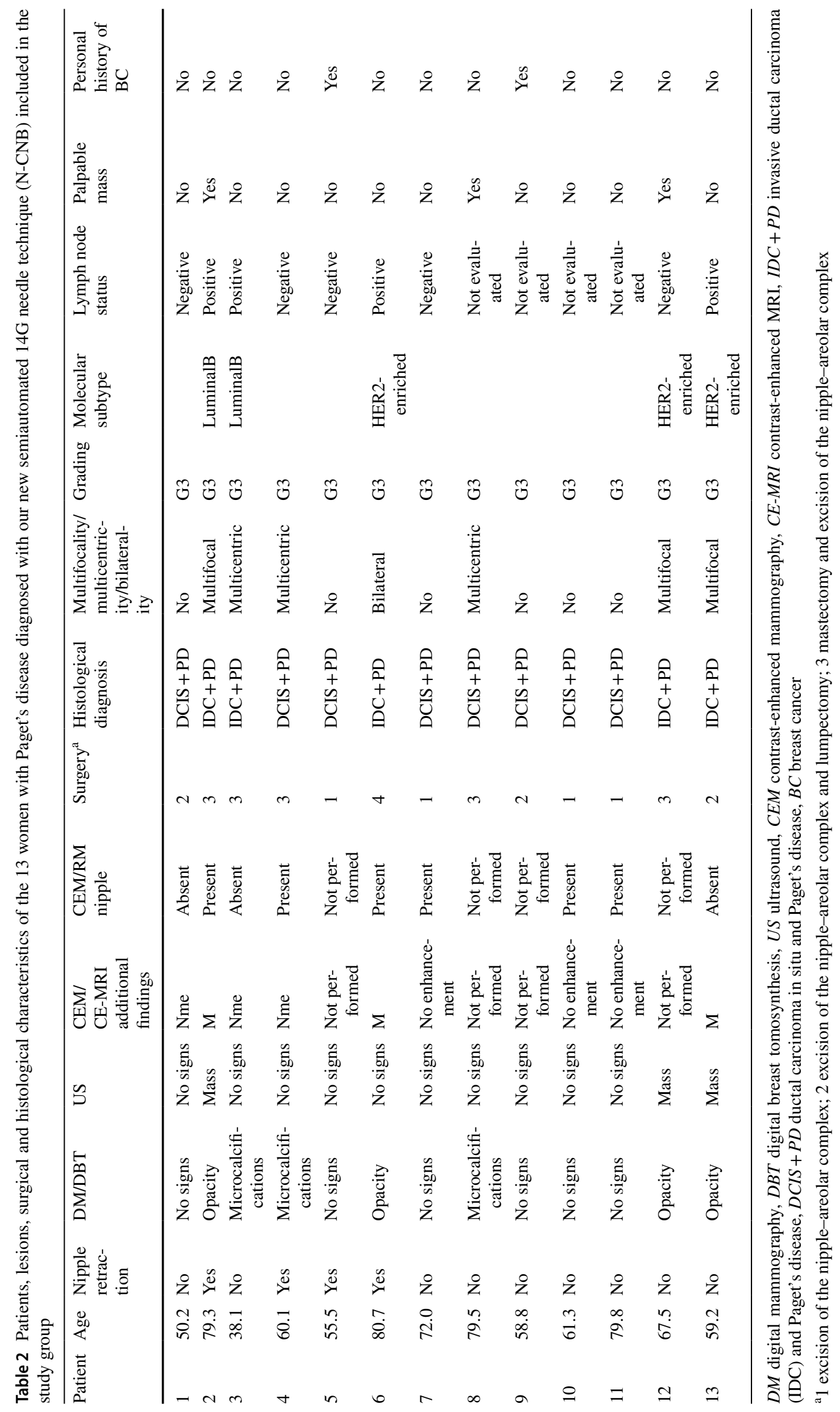


$20 \mathrm{~mm}$ away from the NAC. In four cases, 4/13 (30.8\%) patients refused to perform CEM or CE-MRI.

No significant post-biopsy complications were recorded, only one of the 26 patients (3.8\%) returned to the department an hour after N-CNB due to bleeding, resolved by applying surgical glue and repositioning the patch.

In 6 out of 13 patients, DCIS was diagnosed in the retroareolar lactiferous ducts within the NAC (46.1\%). A secondary lesion (DCIS or invasive carcinoma) elsewhere in the breast, at least $20 \mathrm{~mm}$ away from the NAC, was diagnosed in 7 out of 13 patients $(53.8 \%)$.

In $4 / 13$ (30.8\%) patients, PD was associated with multicentric disease, two (15.4\%) multifocal and one (7.7\%), a bilateral disease.

In 4/13 (30.8\%) patients an excision of the nipple-areolar complex was performed, $4 / 13$ (30.8\%) patients underwent excision of the nipple-areolar complex and lumpectomy, and $5 / 13(38.5 \%)$ mastectomy and excision of the nipple-areolar complex (4 for a multicentric lesion and one for a multifocal lesion in a small breast).

Lymph node metastases were found in 4 patients, all had a CDI associated with PD, two of the Luminal B molecular subtype and two, the HER2 Enriched subtype. Table 2.

A histological analysis of the surgical specimens confirmed the diagnosis of PD obtained with our N-CNB in all 13 cases.

In our series, patients with PD had more frequent nipple retraction $(\rho=0.0407)$ and associated suspicious (i.e., BIRADS 4 and 5) mammographic $(\rho=0.0006)$ findings compared to patients whose N-CNB gave benign results, and the difference was statistically significant. Table 3 .

\section{Discussion}

The aim of our study was to present a new biopsy technique with a semiautomated CNB with a $14 \mathrm{G}$ needle for the diagnosis of PD through a retrospective analysis of our biopsy case series. In our work the diagnosis of PD was obtained in $50 \%$ of patients with clinical alterations of the NAC and suspected PD or with clinical eczema which failed to respond to hydrocortisone, while in $50 \%$ of cases the biopsy resulted in benign skin alterations, i.e., three eczemas, three nipple adenomas and seven benign changes not classified within a specific category but for which the pathologist's report provided a descriptive diagnosis.

All the benign lesions of our N-CNB were confirmed at the clinical-instrumental follow-ups performed in our department, with a follow-up range of 18-49 months.

All the lesions resulting in PD from our N-CNB were then confirmed as such by the histological analysis of the SSs; there were no false positives and the histological diagnosis was obtained in all cases. These results appear to be
Table 3 Clinical, instrumental and histological differences between the two subgroups: benign lesions and Paget's disease lesions, analyzed by applying the Fisher's exact test

\begin{tabular}{|c|c|c|c|c|c|}
\hline & \multicolumn{2}{|c|}{$\begin{array}{l}\text { Paget's } \\
\text { disease }\end{array}$} & \multicolumn{2}{|c|}{$\begin{array}{l}\text { Benign } \\
\text { lesions }\end{array}$} & \multirow[t]{2}{*}{ Fisher's test } \\
\hline & $n$ & $\%$ & $n$ & $\%$ & \\
\hline Overall & 13 & 48.15 & 13 & 51.85 & \\
\hline \multicolumn{6}{|l|}{ Nipple retraction } \\
\hline Present & 4 & 30.77 & 0 & 0.00 & \multirow[t]{2}{*}{$p: \mathbf{0 . 0 4 0 7}$} \\
\hline Absent & 9 & 69.23 & 13 & 100.00 & \\
\hline \multicolumn{6}{|l|}{$\mathrm{DM} / \mathrm{DBT}$} \\
\hline No signs & 6 & 46.15 & 13 & 100.00 & \multirow[t]{3}{*}{$p: \mathbf{0 . 0 0 0 6}$} \\
\hline Opacity & 4 & 30.77 & 0 & 0.00 & \\
\hline Microcalcifications & 3 & 23.08 & 0 & 0.00 & \\
\hline \multicolumn{6}{|l|}{ US } \\
\hline No signs & 10 & 76.92 & 13 & 100.00 & \multirow[t]{2}{*}{$p: 0.0613$} \\
\hline Mass & 3 & 23.08 & 0 & 0.00 & \\
\hline \multicolumn{6}{|l|}{ Histological diagnosis } \\
\hline DCIS and PD & 8 & 61.54 & 0 & 0.00 & \\
\hline IDC and PD & 5 & 38.46 & 0 & 0.00 & \\
\hline Other benign changes & 0 & 0.00 & 7 & 53.84 & \\
\hline Nipple adenoma & 0 & 0.00 & 3 & 23.08 & \\
\hline Eczema & 0 & 0.00 & 3 & 23.08 & \\
\hline
\end{tabular}

Bold values indicate the difference was statistically significant

$D M$ digital mammography, $D B T$ digital breast tomosynthesis, US ultrasound, $I D C+P D$ invasive ductal carcinoma (IDC) and Paget's disease, $D C I S+P D$ Ductal carcinoma in situ and Paget's disease

in contrast with what has been described in the literature on scrape cytology in the diagnosis of PD; scraping, which in fact has a variable false positive results rate in the literature (0-20\%), requires an expert cytologist for the analysis of the material and is painful for the patients $[11-13,18]$. Furthermore, scrape cytology fails to obtain underlying breast tissue $[11,14]$.

Furthermore, from the histopathological analysis of the biopsy samples taken with N-CNB was found that in 11/26 $(42,3 \%)$ of these, in addition to the epidermis and the dermis of the nipple, there was also underlying breast tissue, which allowed the evaluation of the mammary ducts present and a possible diagnosis of breast cancer associated with PD.

There is only one study published in the literature on nipple punch biopsy and two other studies analyzed the use of punch biopsy on the breast [19-21]. Punch biopsy could have non-diagnostic samples, as reported in the study published by Hicks et al. in which 109 nipple punch biopsies were reviewed and one resulted a non-diagnostic sample. Similarly, in the study of Banisadr et al. the authors reported that four punch biopsies failed to determine diagnosis requiring further biopsy or diagnostic excision.

Furthermore, in the study of Modi et al. in three patients the punch biopsy was benign and the final diagnosis was 
instead malignant. With or new biopsy technique, as above mentioned, there were no false positives or negatives and the histological diagnosis was obtained in all cases. The biopsy was also well tolerated by each patient and we had no major complications. Our N-CNB is performed with a semiautomated biopsy gun with a 14-gauge which in our department is usually used for $\mathrm{CNB}$, so there is no need to purchase additional materials and the N-CNB can be easily performed by all doctors who are already used to performing CNB.

The only work in the literature that uses the CNB for the diagnosis of suspicious alterations of the nipple is that of Aryal and colleagues [14]. The authors used a 14G automatic CNB needle (Bard MagnumTM Biopsy instrument) with a selected penetration depth of $22 \mathrm{~mm}$ in 40 patients with suspicious nipple changes. The histological diagnosis was obtained in all 40 cases, similar to our experience.

With our technique we used a semiautomatic needle which, unlike the automatic needle used in Aryal's work, allows for manually opening the cradle, offering the possibility of centering more precisely the area of the nipple to be biopsied. Moreover, with our N-CNB it is not necessary to preset a penetration depth because being able to manually open the cradle means the samples obtained are the entire length of the cradle itself. As reported by the authors of the work described above, also with our N-CNB could be possible to obtain both skin and subcutaneous tissue in which the mammary ducts can be found that could reveal an associated carcinoma. We obtained the underlying breast tissue in 11/26 (42.3\%) of the N-CNB.

Post-biopsy complications are not reported in Aryal's work. Our procedure did not cause any major complications in the patients we studied, i.e., pain, infections. We recorded only one case of post-biopsy bleeding which was resolved with the application of surgical glue and a patch.

The samples obtained through our procedure, as with Aryal's, are similar to those of a normal CNB and could be analyzed in the same way by the pathologist.

In our center we do not perform punch biopsy or scrape cytology, before the introduction of the new biopsy technique presented in the study, in case of clinical of nipple-areola complex changes with suspected PD the patient was sent for incisional biopsy. For this reason the introduction of the N-CNB into our clinical practice has led to significant improvements both in terms of economic savings and above all as less effort for patients and better aesthetic results while maintaining very high diagnostic accuracy.

From November 2016 to November 2019, 5760 CNBs were performed in our department; 2016 of these were malignant breast lesions, of which 13/2016 were PD (0.6\%), in line with the incidence of PD reported in previous studies $[3,4,22]$.

In all cases included in our study, a PD-associated carcinoma was identified; a DCIS was diagnosed in the retroareolar lactiferous ducts within the NAC in six patients with PD, and a secondary lesion (DCIS or invasive carcinoma) elsewhere in the breast at least $20 \mathrm{~mm}$ away from the NAC, was diagnosed in seven patients with PD. Our results appear to be in line with reports in the literature, where in more than $90 \%$ of cases the PD is associated with an underlying in situ or invasive breast carcinoma $[1,4,5,7,23,24]$. This may also explain why the patients with PD in our group showed suspicious mammographic findings more frequently than those in which the histology was benign, and this was a statistically significant difference. To our knowledge there are no published works that have analyzed the clinical, instrumental and histological differences between patients with benign lesions of the CAC and those with PD; therefore, it is not possible to compare our results.

In our patients with $\mathrm{PD}$, mammography revealed additional findings to those of NAC, classified as suspicious (BIRADS 4 or 5) in 7/13 (53.8\%) cases: 3 opacities, one opacity with microcalcifications, and 3 microcalcifications, results that are in line with literature data $[1,25,26]$.

US was found to be useful as part of the initial evaluation in PD patients, especially when mammography was negative as in our work. Ultrasonography identified a suspicious hypoechoic nodule (BI-RADS5) in the contralateral breast of one patient with $\mathrm{PD}$, which, after $\mathrm{CNB}$, proved to be an invasive lobular carcinoma not identified by mammography $[1,27,28]$.

Nine patients in our study group diagnosed with PD performed either CE-MRI or CEM as preoperative staging. CEMRI in the literature has proved useful in the evaluation of patients with PD due to its ability to identify clinically and mammographically occult carcinomas and to differentiate normal from abnormal nipples, and tumors confined to the retroareolar tissue from tumors involving the NAC [3, 26, 29].

To date there are no data in the literature regarding the use of CEM in patients with PD. It would be interesting to analyze the topic more in depth, but only six patients in our group performed CEM and it would have been preferable to also perform CE-MRI in these patients as well to evaluate the diagnostic performance of this new technique. In fact, this represents one of the limitations of our study. The other limitations of the work are the small number of patients and lesions included, the retrospective nature of the study, and a minimum follow-up time of 13 months for some lesions found to be benign with N-CNB, perhaps not long enough to confirm the diagnosis with any great certainty.

At the moment there is still little evidence to recommend one biopsy technique for the histological diagnosis of Paget's disease over another and further research is needed. Our aim is to present a new biopsy technique that in our department has proved to be very accurate and well tolerated, easy, 
without major complications as an initial experience that may perhaps be useful to other departments, to be validated on a greater number of cases.

In conclusion, with the novel technique described above we have been able to obtain an easy, painless, major complication-free and accurate diagnosis of PD of the NAC using a core needle biopsy with a semiautomated 14-gauge needle. A reliable and reproducible technique for diagnosis of PD with CNB which could probably lead to more precise tissue sampling and lower rates of false positives, with a direct impact on the management of individual patients.

Authors' contributions All authors contributed to the study conception and design. Material preparation, data collection and analysis were performed by GB, SB and FA. The first draft of the manuscript was written by GB and all authors commented on previous versions of the manuscript. All authors read and approved the final manuscript.

Funding No funds, grants, or other support was received.

Availability of data and material All data and materials support our published claims and comply with field standards.

\section{Declarations}

Conflicts of interest The authors have no relevant financial or nonfinancial interests to disclose.

Ethics approval This retrospective chart review study involving human participants was in accordance with the ethical standards of the institutional and national research committee and with the 1964 Helsinki Declaration and its later amendments or comparable ethical standards. The Human Investigation Committee (IRB) of AOU Careggi approved this study.

Consent to participate Informed consent was obtained from all individual participants included in the study.

Consent for publication The authors affirm that human research participants provided informed consent for publication of the images in Figs. 1, 2, 3 and 4.

Code availability Not applicable.

\section{References}

1. Sakorafas GH, Blanchard K, Sarr MG et al (2001) Paget's disease of the breast. Cancer Treat Rev 27(1):9-18. https://doi.org/10. 1053/ctrv.2000.0203

2. Chen CY, Sun LM, Anderson BO (2006) Paget disease of the breast: changing patterns of incidence, clinical presentation, and treatment in the U.S. Cancer 107(7):1448-1458. https://doi.org/ $10.1002 /$ cncr. 22137

3. Lim HS, Jeong SJ, Lee JS et al (2011) Paget disease of the breast: mammographic, US, and MR imaging findings with pathologic correlation. Radiographics 31(7):1973-1987. https://doi.org/10. 1148/rg.317115070
4. Sandoval-Leon AC, Drews-Elger K, Gomez-Fernandez CR et al (2013) Paget's disease of the nipple. Breast Cancer Res Treat 141(1):1-12. https://doi.org/10.1007/s10549-013-2661-4 (Epub 2013 Aug 9)

5. Lopes Filho LL, Lopes IM, Lopes LR et al (2015) Mammary and extramammary Paget's disease. An Bras Dermatol 90(2):225-231. https://doi.org/10.1590/abd1806-4841.20153189

6. Kothari AS, Beechey-Newman N, Hamed H et al (2002) Paget disease of the nipple: a multifocal manifestation of higher-risk disease. Cancer 95(1):1-7. https://doi.org/10.1002/cncr.10638

7. Caliskan M, Gatti G, Sosnovskikh I et al (2008) Paget's disease of the breast: the experience of the European Institute of Oncology and review of the literature. Breast Cancer Res Treat 112(3):513521. https://doi.org/10.1007/s10549-007-9880-5 (Epub 2008 Feb 1)

8. Pierce LJ, Haffty BG, Solin LJ et al (1997) The conservative management of Paget's disease of the breast with radiotherapy. Cancer 80(6):1065-1072

9. Marshall JK, Griffith KA, Haffty BG et al (2003) Conservative management of Paget disease of the breast with radiotherapy: 10and 15-year results. Cancer 97(9):2142-2149. https://doi.org/10. 1002/cncr.11337

10. Joseph KA, Ditkoff BA, Estabrook A et al (2007) Therapeutic options for Paget's disease: a single institution long-term followup study. Breast J 13(1):110-111. https://doi.org/10.1111/j.15244741.2006.00381.x

11. Lucarotti ME, Dunn JM, Webb AJ (1994) Scrape cytology in the diagnosis of Paget's disease of the breast. Cytopathology 5(5):301-305. https://doi.org/10.1111/j.1365-2303.1994.tb004 $34 . \mathrm{x}$

12. Raivoherivony ZI, Feron J, Klijanienko J (2019) The utility of nipple scraping in the diagnosis of Paget disease of the breast. Diagn Cytopathol 47(3):249-250. https://doi.org/10.1002/dc. 24033 (Epub 2018 Nov 28)

13. Gupta RK, Simpson J, Dowle C (1996) The role of cytology in the diagnosis of Paget's disease of the nipple. Pathology 28(3):248250. https://doi.org/10.1080/00313029600169084

14. Aryal KR, Lengyel AJ, Purser N, Harrison N, Aluwihare N, Isgar B (2004) Nipple core biopsy for the deformed or scaling nipple. Breast 13(4):350-352. https://doi.org/10.1016/j.breast.2003.12. 007

15. American College of Radiology (2013) ACR BI-RADS® atlas breast imaging reporting and data system. American College of Radiology, Reston

16. Bicchierai G, Amato F, Vanzi B et al (2020) Which clinical, radiological, histological, and molecular parameters are associated with the absence of enhancement of known breast cancers with contrast enhanced digital mammography (CEDM)? Breast 21(54):15-24. https://doi.org/10.1016/j.breast.2020.08.009

17. Bicchierai G, Rigacci L, Miele V et al (2017) Role of core needle biopsy in primary breast lymphoma. Radiol Med 122(9):651-655. https://doi.org/10.1007/s11547-017-0773-3

18. Samarasinghe D, Frost F, Sterrett G et al (1993) Cytological diagnosis of Paget's disease of the nipple by scrape smears: a report of five cases. Diagn Cytopathol 9(3):291-295. https://doi.org/10. $1002 / \mathrm{dc} .2840090309$

19. Hicks G, Brown S, Pepper T et al (2019) The role of nipple punch biopsy. Breast J 25(6):1328-1329. https://doi.org/10.1111/tbj. 13468

20. Modi BN, Machin JT, Ravichandran D (2010) Punch biopsy: a useful adjunct in a rapid diagnosis breast clinic. Breast 19(2):150 151. https://doi.org/10.1016/j.breast.2009.12.007

21. Banisadr S, Ashcroft A, Kumar B, Coveney E (2010) The use of punch biopsy in the diagnosis of cutaneous breast lesions. Eur J Surg Oncol 36(11):1113. https://doi.org/10.1016/j.ejso.2010.08. 026 
22. Karakas C (2011) Paget's disease of the breast. J Carcinog 10:31. https://doi.org/10.4103/1477-3163.90676 (Epub 2011 Dec 8)

23. Yim JH, Wick MR, Philpott GW et al (1997) Underlying pathology in mammary Paget's disease. Ann Surg Oncol 4(4):287-292. https://doi.org/10.1007/BF02303576

24. Fu W, Mittel VK, Young SC (2001) Paget disease of the breast: analysis of 41 patients. Am J Clin Oncol 24(4):397-400. https:// doi.org/10.1097/00000421-200108000-00019

25. Sawyer RH, Asbury DL (1994) Mammographic appearances in Paget's disease of the breast. Clin Radiol 49(3):185-188. https:// doi.org/10.1016/s0009-9260(05)81773-8

26. Ikeda DM, Helvie MA, Frank TS et al (1993) Paget disease of the nipple: radiologic-pathologic correlation. Radiology 189(1):8994. https://doi.org/10.1148/radiology.189.1.8396786

27. Kamio T, Hamano K, Kameoka S, Kimura T (1996) Ultrasonographic diagnosis of breast cancer with intraductal spreading of cancer cells. Nihon Geka Gakkai Zasshi 97(5):338-342 ((Japanese))

28. Yang WT, King W, Metreweli C (1997) Clinically and mammographically occult invasive ductal carcinoma diagnosed by ultrasound: the focally dilated duct. Australas Radiol 41(1):73-75. https://doi.org/10.1111/j.1440-1673.1997.tb00476.x

29. Friedman EP, Hall-Craggs MA, Mumtaz H, Schneidau A (1997) Breast MR and the appearance of the normal and abnormal nipple. Clin Radiol 52(11):854-861. https://doi.org/10.1016/s00099260(97)80081-5

Publisher's Note Springer Nature remains neutral with regard to jurisdictional claims in published maps and institutional affiliations. 